\title{
Е.В. Буценко
}

\section{ПРАКТИКА ПРИМЕНЕНИЯ СЕТЕВОГО ЭКОНОМИКО-МАТЕМАТИЧЕСКОГО МОДЕЛИРОВАНИЯ ПРОЦЕССА ИНВЕСТИЦИОННОГО ПРОЕКТИРОВАНИЯ}

\begin{abstract}
В статье рассматриваются вопросы экономико-математического моделирования процесса управления инвестиционным проектированием для хозяйствующего субъекта. Для разработки экономико-математической модели процесса управления инвестиичинным проектированием предлагается использовать методы сетевого планирования и управления. Предложенная в работе соответствующцая рассматриваемому процессу сетевая модель позволяет реализовать эффективную организацию работ по инвестиционному проектированию. В статье приведен реальный пример применения предлагаемой сетевой модели для разработки инвестиционного проекта по открытию компании для этапа ввода в эксплуатацию производственного помещения компании пищевой отрасли. Для данного примера в работе сделан анализ, упорядочение и кодирование работ, рассчитаны параметры сетевого моделирования, построен соответствующий сетевой график, календарный график работ для рассматриваемого процесса инвестиционного проектирования.

Ключевые слова: инвестиционное проектирование, сетевое моделирование, параметры сетевой модели, календарный график.
\end{abstract}

Методы сетевого и календарного планирования и управления известны давно. Они появились в 40-50-х гг. ХХ в. в СССР и США, независимо друг от друга, для решения крупных военных и технических задач. В США метод календарного планирования называется ПЕРТ. Этот метод позволяет любой крупный проект представить в виде последовательности элементарных работ и разрабатывать эффективные процедуры наглядного отображения проекта и решения различных задач в рамках этого проекта.

В настоящее время сетевые методы широко используются в транспортной сфере, отрасли связи, строительстве и других областях жизнедеятельности человека.

Например, в работе Т.М. Зеленской с соавт. предлагается применять методы сетевого планирования и управления в сельскохозяйственном производстве [1]. В статье М.Ю. Иванова рассмотрены базовые математические вопросы построения сетевых графиков и методы сетевого планирования и управления в сфере банковских услуг [2]. В.Г. Киселевым описаны сетевые модели управления в животноводческой отрасли [3]. Д.О. Приходько в качестве инновационного инструмента моделирования процессов строительной организации предложил сетевую модель с параметрами нового содержания [4].

Научной гипотезой данной работы является новое применение известных методов сетевого планирования и управления для детерминированного экономико-математического моделирования процессов инвестиционного проек- 
тирования для хозяйствующего субъекта. Сферой применения методов сетевого моделирования выбрана пищевая промышленность, так как большое количество инвестиций малого бизнеса в современных условиях деловой среды связано именно с ней.

Определим, что экономико-математической моделью будет сетевая модель, соответствующая процессам инвестиционного проектирования. Для оптимизации процесса управления инвестиционным проектированием на основе сетевых методов необходимо привести описание экономикоматематической сетевой модели.

Выделим последовательность основных блоков работ инвестиционного проектирования [5]:

1) сбор исходных данных для реализации процесса инвестиционного проектирования (блок работ $\mathrm{A}_{1}$ );

2) маркетинговый анализ проектов (блок работ $\mathrm{B}_{2}$ );

3) финансовый анализ (расчет финансовых показателей) проектов (блок работ $\mathrm{C}_{3}$ );

4) анализ чувствительности (влияния рисков на проекты) (блок работ $\mathrm{D}_{2}$ );

5) построение финансовой модели инвестиционного проектирования (блок работ $\mathrm{E}_{2}$ );

6) формирование альтернативных вариантов условий реализации проектов (блок работ $\mathrm{F}_{2}$ );

7) анализ полученных результатов и выбор оптимального инвестиционного проекта (блок работ $\mathrm{G}_{2}$ ).

Каждый из отмеченных выше блоков работ, определяющих структуру рассматриваемого процесса, образует многоуровневую сетевую модель процесса инвестиционного проектирования.

Для формирования общей методики инвестиционного проектирования с применением экономико-математического моделирования приведем основные работы каждого из выделенных блоков работ. Их кодирование соответствует указанной выше последовательности основных этапов инвестиционного проектирования и состоит из букв и цифр, где 1-я цифра соответствует номеру блока работ, 2-я - номеру работы в блоке: $\mathrm{A}_{11}$ - принятие решения об инвестировании; $\mathrm{A}_{12}$ - расчет расходов на строительство; $\mathrm{A}_{13}$ - расчет расходов на НИОКР; $\mathrm{A}_{14}$ - расчет затрат на производство и реализацию продукции/услуг; $\mathrm{A}_{15}$ - расчет расходов на амортизацию; $\mathrm{A}_{16}$ - расчет объема инвестиций в проект; $\mathrm{A}_{17}-$ расчет приемлемой процентной ставки за кредит; $\mathrm{A}_{18}-$ получение начальной исходной информации по инвестированию; $\mathrm{B}_{21}$ - формирование исходных данных для маркетингового анализа; $\mathrm{B}_{22}$ - исследование рынка; $\mathrm{B}_{23}$ - оценка состояния конкуренции и других внешних факторов; $\mathrm{B}_{24}$ - анализ рыночных цен на продукцию/услуги; $\mathrm{B}_{25}$ - формирование стратегии маркетинга; $\mathrm{B}_{26}$ - формирование прогноза объема продаж/услуг; $\mathrm{B}_{27}-$ маркетинговый анализ; $\mathrm{B}_{28}$ - формирование плана маркетинга; $\mathrm{C}_{31}$ - отбор данных для расчета финансовых показателей инвестиционного проектирования; $\mathrm{C}_{32}$ - анализ структуры активов и пассивов баланса проекта, структуры распределения прибыли и др.; $\mathrm{C}_{33}$ - анализ динамики финансовых показателей проектов; $\mathrm{C}_{34}$ - определение финансовых показателей проекта; $\mathrm{C}_{35}$ - pacчет коэффициентов рентабельности; $\mathrm{C}_{36}$ - расчет коэффициентов оборачи- 
ваемости; $\mathrm{C}_{37}$ - расчет коэффициентов финансовой устойчивости; $\mathrm{C}_{38}-$ расчет коэффициентов ликвидности; $\mathrm{C}_{39}$ - анализ финансовых показателей проекта; $\mathrm{C}_{310}$ - прогноз финансовых результатов от реализации проекта; $\mathrm{D}_{41}-$ принятие решения о необходимости проведения анализа чувствительности и формирование соответствующей исходной информации; $\mathrm{D}_{42}$ - выбор результирующих показателей эффективности инвестиционного проекта; $\mathrm{D}_{43}$ - формирование факторов неопределенности; $\mathrm{D}_{44}$ - определение предельных значений факторов неопределенности; $\mathrm{D}_{45}$ - расчет результирующих показателей эффективности проекта для всех допустимых значений факторов неопределенности; $\mathrm{D}_{46}$ - анализ полученных результатов (заключение о наиболее критических факторах инвестиционного проекта); $\mathrm{E}_{51}$ - установление целей создания модели, отбор выходных данных из блоков финансового анализа и анализа чувствительности; $\mathrm{E}_{52}$ - формирование критериев качества, оценивающих результаты инвестиционного проектирования; $\mathrm{E}_{53}$ - выбор наиболее приемлемых схем инвестирования средств и источников финансирования для реализации проекта; $\mathrm{F}_{61}$ - определение возможных вариантов проектов на основе полученной на предыдущих этапах информации; $\mathrm{F}_{62}$ - формирование вариантов условий реализации проектов; $\mathrm{G}_{71}$ - формирование конкретных показателей оценки эффективности инвестиционных проектов; $\mathrm{G}_{72}$ - расчет значений сформированных показателей эффективности для всех инвестиционных проектов; $\mathrm{G}_{73}$ - сравнительный анализ и выбор наилучших значений показателей эффективности и соответствующего им инвестиционного проекта/проектов; $\mathrm{G}_{74}$ - описание и наглядное представление всех собранных и полученных расчетных данных по выбранному для инвестирования проекту/проектам.

На рис. 1 показан сетевой график, соответствующий выделенным работам процесса инвестиционного проектирования.

Таким образом, сформирована общая сетевая модель процесса инвестиционного проектирования, которую можно использовать для оптимизации управления данным процессом.

В качестве демонстрации действий по построению сетевой модели приведем один из этапов проектирования, связанный с расчетом затрат для определения финансовых показателей проекта. Для данного примера в работе проведем цикл необходимых действий по анализу, упорядочению и кодированию выделенных работ, построим соответствующий сетевой график, рассчитаем параметры сетевого моделирования и построим календарный график.

Приведем последовательность действий сетевого моделирования:

1) определение необходимых действий/работ, соответствующих процесcy;

2) анализ, упорядочение и кодирование выделенных работ;

3) построение соответствующего сетевого графика;

4) расчет параметров сетевого моделирования;

5) построение календарного графика. 


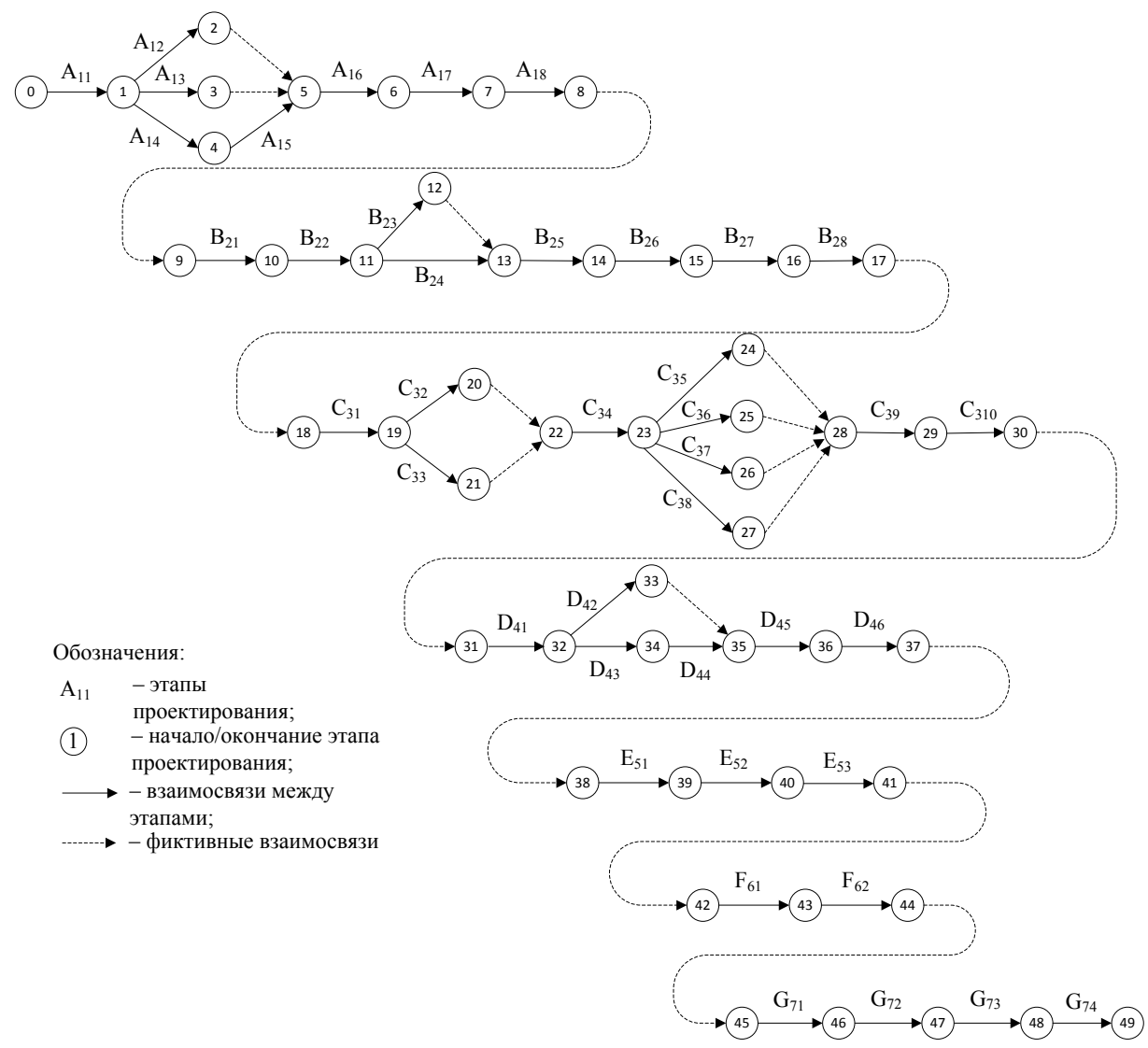

Рис. 1. Сетевой график модели инвестиционного проектирования

Будем рассматривать применение методов сетевого планирования и управления при разработке инвестиционного проекта по открытию компании в пищевой отрасли по производству замороженных продуктов в г. Екатеринбурге для этапа ввода в эксплуатацию производственного помещения.

Исходная информация в виде комплекса работ с указанием кодировки и продолжительности их выполнения представлена в табл. 1.

Данные в прографке 2 табл. 1 получаем после проведения анализа и упорядочения работ этапа ввода в эксплуатацию. Этот этап является наиболее сложным и трудоемким, так как требует от аналитика большого опыта и знаний.

На основе информации из табл. 1 строим сетевой график, задающий структуру и взаимосвязи рассматриваемого этапа проекта, представленный на рис. 2.

Из анализа построенного графика видно, что он имеет коэффициент сложности, равный 2 , что показывает достаточно высокий уровень определения взаимосвязей и работ проекта [6]. 


\section{Таблица 1. Исходные данные ${ }^{1}$}

\begin{tabular}{|c|c|c|c|}
\hline $\begin{array}{l}\text { № } \\
\Pi / \Pi\end{array}$ & $\begin{array}{c}\text { Код } \\
\text { работы }\end{array}$ & Содержание работы & $\begin{array}{l}\text { Продолжи- } \\
\text { тельность, } \\
\text { дни }\end{array}$ \\
\hline 1 & $\mathrm{C}_{1}$ & $\begin{array}{l}\text { Представление нотариально заверенной копии учредительных } \\
\text { документов компании (Устав, Свидетельство о регистрации } \\
\text { предприятия, Учредительный договор, Свидетельство о поста- } \\
\text { новке на учет в налоговой инспекции, справка о присвоении } \\
\text { кодов из Госкомстата) }\end{array}$ & 1 \\
\hline 2 & $\mathrm{C}_{2}$ & Представление договора аренды (субаренды) & 1 \\
\hline 3 & $\mathrm{C}_{3}$ & $\begin{array}{l}\text { Представление свидетельства собственника на арендуемое } \\
\text { помещение }\end{array}$ & 1 \\
\hline 4 & $\mathrm{C}_{4}$ & Представление планов БТИ на арендуемое помещение & 14 \\
\hline 5 & $\mathrm{C}_{5}$ & $\begin{array}{l}\text { Представление Справки об отсутствии задолженности компа- } \\
\text { нии по уплате налогов и сборов из налоговой инспекции }\end{array}$ & 4 \\
\hline 6 & $\mathrm{C}_{6}$ & $\begin{array}{l}\text { Регистрация кассовых аппаратов. Карточки регистрации кон- } \\
\text { трольно-кассовых аппаратов в налоговой инспекции района }\end{array}$ & 14 \\
\hline 7 & $\mathrm{C}_{22}$ & Санитарно-эпидемиологическая экспертиза объекта & 10 \\
\hline 8 & $\mathrm{C}_{23}$ & $\begin{array}{l}\text { Получение санитарно-эпидемиологического заключения } \\
\text { ЦГСЭН о соответствии санитарным правилам, определяющих } \\
\text { потенциальную опасность для здоровья человека, видов дея- } \\
\text { тельности, работ, услуг }\end{array}$ & 5 \\
\hline 9 & $\mathrm{C}_{16}$ & $\begin{array}{l}\text { Получение заключения УГПС административного округа о } \\
\text { соответствии объекта противопожарным требованиям }\end{array}$ & 10 \\
\hline 10 & $\mathrm{C}_{17}$ & $\begin{array}{l}\text { Получение заключения ОВД о наличии пожарно-охранной и } \\
\text { охранной сигнализаций для лицензирования }\end{array}$ & 10 \\
\hline 11 & $\mathrm{C}_{7}$ & $\begin{array}{l}\text { Проведение замеров уровней шумов от холодильного и техно- } \\
\text { логического оборудования с выдачей протоколов }\end{array}$ & 10 \\
\hline 12 & $\mathrm{C}_{8}$ & $\begin{array}{l}\text { Проведение исследований питьевой воды, воздуха с выдачей } \\
\text { протоколов }\end{array}$ & 10 \\
\hline 13 & $\mathrm{C}_{9}$ & $\begin{array}{l}\text { Заключение договора на проведение дезинфекции и дератиза- } \\
\text { ции }\end{array}$ & 5 \\
\hline 14 & $\mathrm{C}_{10}$ & Заключение договора на вывоз мусора & 5 \\
\hline 15 & $\mathrm{C}_{11}$ & $\begin{array}{l}\text { Представление списка сотрудников компании с данными мед- } \\
\text { осмотров, медицинскими книжками }\end{array}$ & 4 \\
\hline 16 & $\mathrm{C}_{12}$ & Представление откорректированного ассортиментного перечня & 5 \\
\hline 17 & $\mathrm{C}_{13}$ & Согласование ассортиментного перечня в ЦГСЭН & 5 \\
\hline 18 & $\mathrm{C}_{14}$ & $\begin{array}{l}\text { Разработка и представление программы производственного } \\
\text { контроля }\end{array}$ & 4 \\
\hline 19 & $\mathrm{C}_{15}$ & $\begin{array}{l}\text { Заключение договора на проведение лабораторных испытаний } \\
\text { по производственному контролю }\end{array}$ & 4 \\
\hline 20 & $\mathrm{C}_{18}$ & $\begin{array}{l}\text { Оформление журналов (по технике безопасности, бракераж- } \\
\text { ный, аварийных ситуаций, списания продуктов, санитарный } \\
\text { журнал и другие необходимые для работы компании) }\end{array}$ & 5 \\
\hline 21 & $\mathrm{C}_{19}$ & Составление штатного расписания & 10 \\
\hline 22 & $\mathrm{C}_{20}$ & Представление выписки из приказа о назначении директора & 1 \\
\hline 23 & $\mathrm{C}_{21}$ & $\begin{array}{l}\text { Получение согласования на открытие компании в ОАТИ адми- } \\
\text { нистративного округа }\end{array}$ & 14 \\
\hline 24 & $\mathrm{C}_{24}$ & Получение согласования на открытие компании в ЦГСЭН & 14 \\
\hline 25 & $\mathrm{C}_{26}$ & Получение согласования на открытие компании в УГПС & 14 \\
\hline 26 & $\mathrm{C}_{27}$ & Получение и согласование паспорта на рекламную вывеску & 7 \\
\hline 27 & $\mathrm{C}_{28}$ & Получение сертификата соответствия выпускаемой продукции & 3 \\
\hline 28 & $\mathrm{C}_{29}$ & $\begin{array}{l}\text { Укомплектование компании нормативной документацией (Закон о } \\
\text { защите прав потребителя, Санитарные нормы и правила и др.) }\end{array}$ & 2 \\
\hline 29 & $\mathrm{C}_{25}$ & Разработка и утверждение плана эвакуации людей при пожаре & 7 \\
\hline 30 & $\mathrm{C}_{30}$ & Обеспечение работы грузового лифта & 2 \\
\hline
\end{tabular}

${ }^{1}$ Кодирование работ не продолжает вышерассмотренную последовательность нумерации работ в блоке, так как приводится усеченный пример одного из подуровней многоуровневой сетевой модели инвестиционного проектирования. 


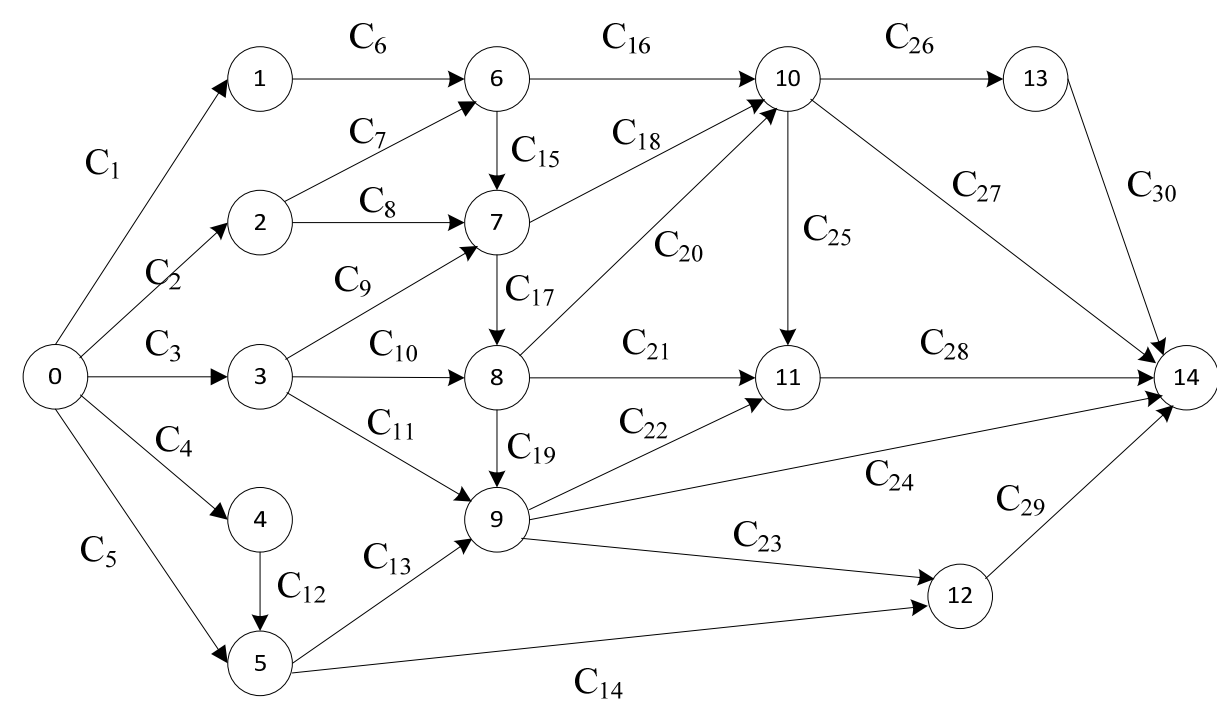

Рис. 2. Сетевой график, отражающий работы и их взаимосвязи в проекте

Таким образом, в виде построенного графика мы выполнили третий этап последовательности действий сетевого моделирования и переходим к четвертому этапу, который включает расчет параметров сети.

Расчет параметров сетевого моделирования возможен двумя способами: аналитическим - в виде построения таблицы необходимых для анализа параметров и графическим, который более наглядно отражает расчеты. Покажем на втором способе расчет параметров построенного сетевого графика модели.

Приведем обозначения для параметров модели, которые будут отображены в секторах событий-кругов на рис. 3 .

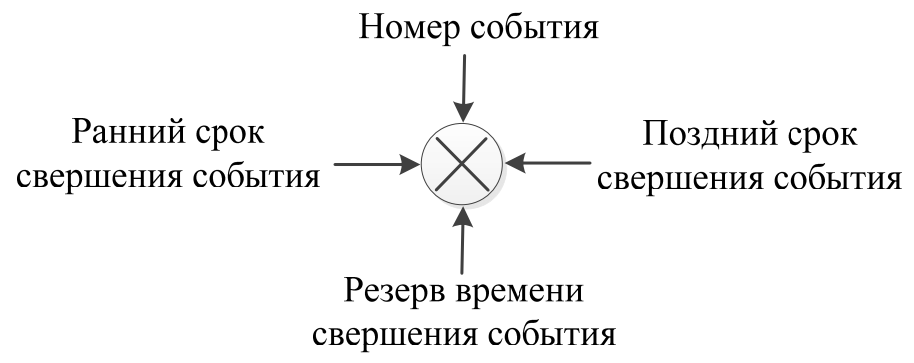

Рис. 3. Расположение параметров сетевой модели на графике

На рис. 4 отображен сетевой график с рассчитанными параметрами модели. 


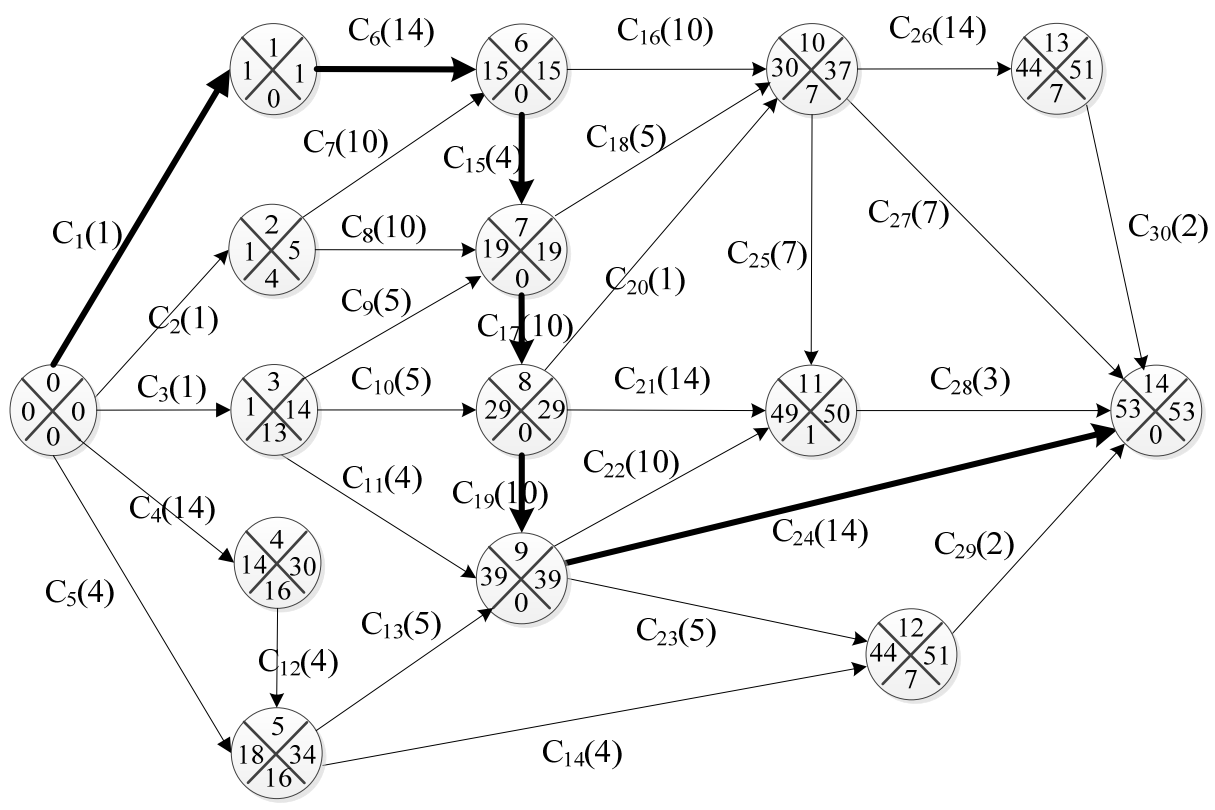

Рис. 4. Параметры сетевого графика построенной модели

Расчет параметров начинают с определения ранних сроков наступления событий, затем рассчитывают поздние сроки завершения событий, а также определяют резервы времени свершения событий и находят критический путь модели.

Все параметры мы рассчитали, определим критическое или оптимальное время для рассматриваемого проекта, т.е. наименьший период времени, необходимый для реализации проекта в целом. Завершающее 14-е событие может свершиться лишь на 53-й день от начала реализации работ проекта, следовательно, 53 дня - минимальное время, за которое может быть выполнен весь проект в целом, т.е. в нашем примере критический путь состоит из операций: $\mathrm{C}_{1} \rightarrow \mathrm{C}_{6} \rightarrow \mathrm{C}_{15} \rightarrow \mathrm{C}_{17} \rightarrow \mathrm{C}_{19} \rightarrow \mathrm{C}_{24}$, а критическое время составляет 53 дня.

На рис. 4 критический путь выделен полужирными линиями. Таким образом, мы получили, что период времени, необходимый для реализации рассматриваемого проекта в целом, уменьшился на $201-53=148$ дней, т.е. на $26 \%$.

Нахождение критического пути позволяет сосредоточить на соответствующих работах дополнительные средства и, сократив срок исполнения проекта в целом, ускорить достижение общих целей реализации проекта.

Работы, входящие в критический путь, не имеют резерва времени для их выполнения, и в случае изменения их длительности, меняются сроки исполнения всего проекта в сторону увеличения времени, необходимого для его реализации. В связи с этим при выполнении проекта критические работы требуют большего контроля, в частности, своевременного выявления проблем и рисков, влияющих на сроки их выполнения и, следовательно, на сроки 
выполнения проекта в целом. В процессе выполнения проекта критический путь может меняться, так как при изменении длительности работ некоторые из них могут оказаться на критическом пути.

Полученные данные для возможных календарных сроков исполнения операций можно отобразить на графике. По оси $O Y$ расположим работы проекта. Ось $O X$ - ось времени, т.е. длительность выполнения работ в днях. Длина отрезков соответствует возможной (максимальной) продолжительности длины работы. Критические работы располагаются друг за другом без временных сдвигов (рис. 5).

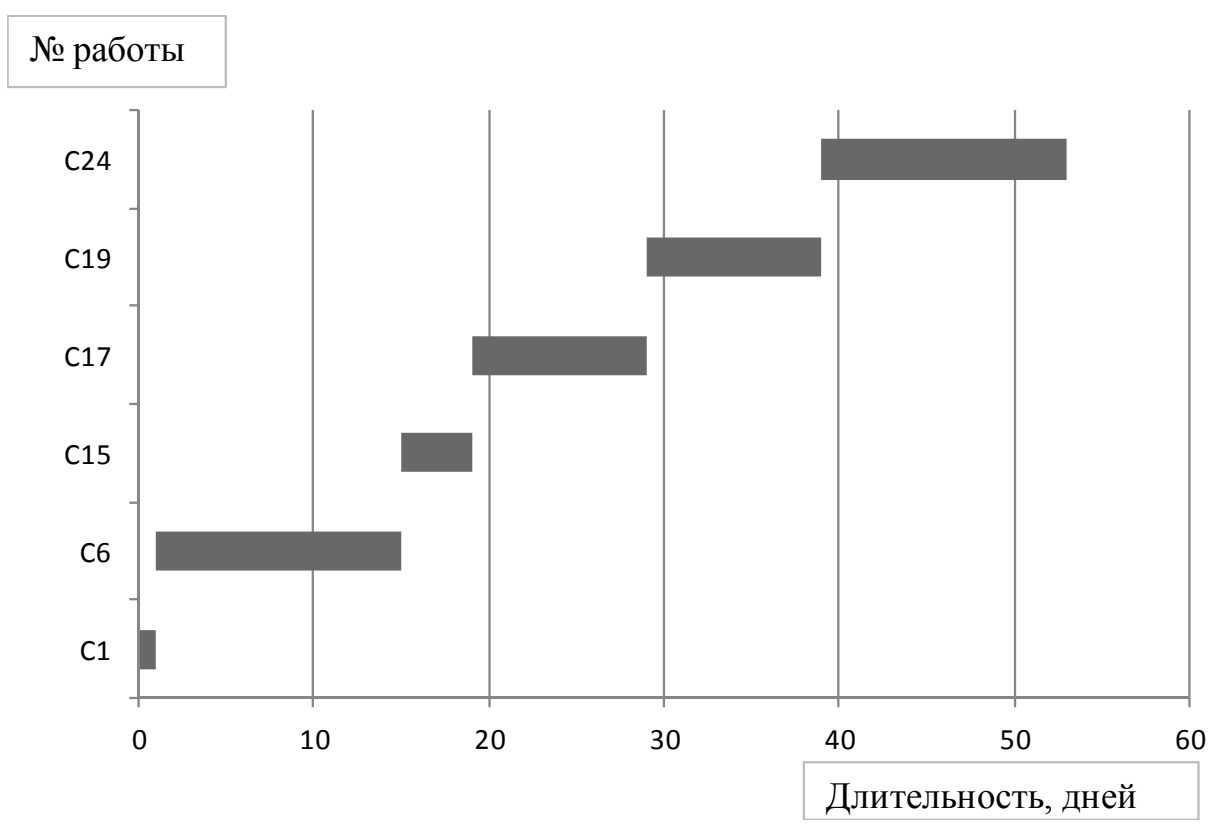

Рис. 5. Диаграмма с временным графиком

На графике показаны сроки выполнения всех критических работ построенной сетевой модели.

Выше применение сетевого моделирования для инвестиционного проектирования касалось только одного параметра времени - длительности работ. Далее проведем оптимизацию сформированной сетевой модели инвестиционного проектирования для этой же компании по производству замороженных продуктов и аналогичного этапа ввода в эксплуатацию производственного помещения по параметру стоимости.

Каждая $(i, j)$-тая работа характеризуется продолжительностью $t_{i j}$, которая может находиться в пределах:

$$
a_{i j} \leq t_{i j} \leq b_{i j}
$$


где $a_{i j}$ - минимально возможная продолжительность $(i, j)$-той работы, которую только можно осуществить в условиях реализации проекта; $b_{i j}$ - нормальная продолжительность выполнения $(i, j)$-той работы.

При этом стоимость работы $c_{i j}$ заключена в границах от $c_{\min }$ (при нормальной продолжительности работы) до $c_{\max }$ (при минимальной продолжительности работы).

Затраты, необходимые на ускорение $(i, j)$-той работы (по сравнению с нормальной ее продолжительностью), отнесенные на единицу времени, рассчитываются по формуле

$$
h_{i j}=\frac{c_{\max i j}-c_{\min i j}}{b_{i j}-a_{i j}},
$$

где $h_{i j}$ - коэффициент затрат на ускорение $(i, j)$-той работы.

Стоимость выполнения проекта до оптимизации определяется как

$$
C=\sum_{i j} c_{i j}
$$

Стоимость выполнения проекта после оптимизации уменьшится на величину

$$
\Delta C=\sum_{i j} \Delta c_{i j}=\sum_{i j}\left(b_{i j}-t_{i j}\right) \cdot h_{i j}
$$

Граничные значения длительностей работ $a_{i j}$ и $b_{i j}$, их стоимости $c_{i j}$, коэффициенты затрат на ускорение работ $h_{i j}$ приведены в табл. 2. Свободные резервы времени работ для критических работ в сетевой модели данного проекта равны нулю. Результаты оптимизации сетевой модели по параметру стоимости приведем в табл. 2 .

В табл. 2 представлены параметры только тех работ, которые имеют свободные резервы времени. Критические работы не приведены в ней, и для них расчеты не требуются, так как у них отсутствуют свободные резервы времени (в табл. 2 напротив значений критических работ стоят прочерки). Расчет коэффициента затрат на ускорение проводится по формуле (2), для примера рассчитаем его для работы $\mathrm{C}_{4}: h_{i j}=\frac{3000-2000}{21-7}=71,4$, а $\Delta C$ рассчитывается по формуле (4).

Стоимость первоначального варианта реализации проекта на основе сформированной сетевой модели равна сумме стоимостей всех определяющих его работ: $C=\sum_{i j} c_{i j}=58704$ руб. Стоимость реализации проекта после оптимизации по показателю стоимости равна $C^{\prime}=C-\Delta C=58704$ $23621,13=35$ 082,87 руб., т.е. стоимость уменьшилась на 40\%. 
Таблица 2. Оптимизация сетевой модели инвестиционного проектирования по параметру стоимости

\begin{tabular}{|c|c|c|c|c|c|c|c|c|c|}
\hline \multirow[t]{2}{*}{$\begin{array}{c}\text { Код } \\
\text { работы }\end{array}$} & \multirow[t]{2}{*}{$\begin{array}{c}\text { Работа } \\
(i, j)\end{array}$} & \multicolumn{3}{|c|}{$\begin{array}{c}\text { Продолжительность } \\
\text { работы, дни }\end{array}$} & \multicolumn{3}{|c|}{$\begin{array}{c}\text { Стоимость работы, } \\
\text { тыс. руб. }\end{array}$} & \multirow[t]{2}{*}{$h_{i j}$} & \multirow[t]{2}{*}{$\Delta C$} \\
\hline & & $a_{i j}$ & $t_{i j}$ & $b_{i j}$ & $c_{\min }$ & $c_{i j}$ & $c_{\max }$ & & \\
\hline $\mathrm{C}_{1}$ & $(0,1)$ & 1 & 1 & 20 & 50 & 100 & 200 & - & - \\
\hline $\mathrm{C}_{2}$ & $(0,2)$ & 1 & 1 & 40 & 50 & 100 & 200 & 3,8 & 148,2 \\
\hline $\mathrm{C}_{3}$ & $(0,3)$ & 1 & 1 & 9 & 1 & 2 & 3 & 0,25 & 2 \\
\hline $\mathrm{C}_{4}$ & $(0,4)$ & 7 & 14 & 21 & 2000 & 2500 & 3000 & 71,4 & 499,8 \\
\hline $\mathrm{C}_{5}$ & $(0,5)$ & 1 & 4 & 7 & 150 & 200 & 250 & 16,6 & \\
\hline $\mathrm{C}_{6}$ & $(1,6)$ & 1 & 14 & 21 & 1000 & 1200 & 1500 & - & - \\
\hline $\mathrm{C}_{7}$ & $(2,6)$ & 5 & 10 & 14 & 1500 & 1700 & 3400 & 211,1 & 844,4 \\
\hline $\mathrm{C}_{8}$ & $(2,7)$ & 7 & 10 & 14 & 1700 & 2000 & 5000 & 471,4 & 1885,6 \\
\hline $\mathrm{C}_{9}$ & $(3,7)$ & 3 & 5 & 7 & 1500 & 2000 & 3000 & 375 & 750 \\
\hline $\mathrm{C}_{10}$ & $(3,8)$ & 3 & 5 & 7 & 2000 & 4000 & 6000 & 1000 & 2000 \\
\hline $\mathrm{C}_{11}$ & $(3,9)$ & 3 & 4 & 7 & 1000 & 2000 & 3000 & 500 & 1500 \\
\hline $\mathrm{C}_{12}$ & $(4,5)$ & 3 & 5 & 7 & 1000 & 2000 & 3000 & 500 & 1000 \\
\hline $\mathrm{C}_{13}$ & $(5,9)$ & 3 & 5 & 7 & 500 & 1000 & 2000 & 375 & 750 \\
\hline $\mathrm{C}_{14}$ & $(5,12)$ & 3 & 4 & 7 & 2000 & 3000 & 5000 & 750 & 2250 \\
\hline$\overline{C_{15}}$ & $(6,7)$ & 2 & 4 & 7 & 2000 & 3000 & 4000 & - & - \\
\hline$\overline{C_{16}}$ & $(6,10)$ & 7 & 10 & 14 & 500 & 700 & 1000 & 71,4 & 285,6 \\
\hline$\overline{C_{17}}$ & $(7,8)$ & 7 & 10 & 14 & 700 & 1000 & 1500 & - & - \\
\hline$\overline{C_{18}}$ & $(7,10)$ & 3 & 5 & 6 & 2000 & 2500 & 3000 & 333,33 & 333,33 \\
\hline $\mathrm{C}_{19}$ & $(8,9)$ & 5 & 10 & 14 & 100 & 200 & 300 & - & - \\
\hline $\mathrm{C}_{20}$ & $(8,10)$ & 1 & 1 & 2 & 1 & 2 & 3 & 2 & 2 \\
\hline $\mathrm{C}_{21}$ & $(8,11)$ & 7 & 14 & 21 & 700 & 1000 & 1500 & 57,1 & 399,7 \\
\hline $\mathrm{C}_{22}$ & $(9,11)$ & 7 & 10 & 14 & 7000 & 10000 & 13000 & 857,1 & 3428,4 \\
\hline $\mathrm{C}_{23}$ & $(9,12)$ & 3 & 5 & 7 & 1000 & 2000 & 3000 & 500 & 1000 \\
\hline $\mathrm{C}_{24}$ & $(9,14)$ & 10 & 14 & 21 & 700 & 1000 & 1500 & - & - \\
\hline $\mathrm{C}_{25}$ & $(10,11)$ & 5 & 7 & 10 & 2000 & 3500 & 5000 & 600 & 1800 \\
\hline $\mathrm{C}_{26}$ & $(10,13)$ & 10 & 14 & 21 & 700 & 1000 & 1500 & 72,7 & 508,9 \\
\hline $\mathrm{C}_{27}$ & $(10,14)$ & 5 & 7 & 10 & 1000 & 1500 & 2000 & 200 & 600 \\
\hline $\mathrm{C}_{28}$ & $(11,14)$ & 2 & 3 & 5 & 4500 & 6000 & 8000 & 1166,6 & 2333,2 \\
\hline $\mathrm{C}_{29}$ & $(12,14)$ & 1 & 2 & 3 & 1000 & 1500 & 2000 & 500 & 500 \\
\hline $\mathrm{C}_{30}$ & $(13,14)$ & 1 & 2 & 4 & 1800 & 2000 & 3000 & 400 & 800 \\
\hline Итого & & & & & & 58704 & - & - & 23621,13 \\
\hline
\end{tabular}

Таким образом, в результате оптимизации сетевой модели для рассматриваемого этапа инвестиционного проекта сформирован план работ, позволяющий выполнить весь комплекс необходимых работ за период времени 148 дней при минимальной его стоимости 35 тыс. руб. Если требуется ускорение выполнения данного этапа, то это, естественно, отразится на его стоимости, т.е. она увеличится.

В заключение отметим, что в статье рассмотрено новое применение методов сетевого моделирования с целью оптимизации процесса инвестиционного проектирования для хозяйствующего субъекта. Описана общая методология применения данных методов к процессу инвестиционного проектирования, а также приведен практический пример построения сетевой экономико-математической модели для этапа ввода в эксплуатацию производственного помещения компании по производству замороженных продуктов. Использование предлагаемой методики как инструментария для реализации процесса инвестиционного проектирования позволит хозяйствующему субъекту повысить свой экономический потенциал и конкурентоспособность. Се- 
тевое моделирование процесса оптимизации инвестиционного проектирования дает возможность последовательно принимать важные управленческие решения. При оптимизации работ инвестиционного проектирования для конкретной компании повышается эффективность ее функционирования и позиционирования на рынке.

Также отметим, что основное преимущество предлагаемого в данной работе сетевого экономико-математического моделирования для реализации процесса инвестиционного проектирования перед традиционными методами его формирования состоит в том, что оно позволяет оптимизировать достижение поставленных целей при реализации конкретного проекта в соответствии с выбранными критериями его качества, т.е. оптимизировать управление процессом инвестиционного проектирования.

Последовательное выполнение функций инвестиционного проектирования, заложенное в методах сетевого планирования и управления и основанное на информационной поддержке инвестиционных решений, позволяет оптимально управлять инвестиционными проектами на всех стадиях их реализации. Эффективное планирование и управление процессом инвестиционного проектирования сокращает общую продолжительность выполнения всего комплекса необходимых работ, уменьшая число сбоев из-за несогласованности используемых ресурсов, а за счет снижения суммарной потребности в ресурсах соответственно снижается общая стоимость проекта.

Таким образом, предлагаемое в статье новое использование сетевого моделирования в качестве инструментария для решения задач управления процессом инвестиционного проектирования в деятельности хозяйствующего субъекта является эффективным способом оптимизации управления процессом инвестиционного проектирования.

\section{Лuтература}

1. Зеленская Т.М., Ванжула Д.В., Ковалева К.А. Применение методов сетевого планирования и управления в сельскохозяйственном производстве // Политематический сетевой электрон. науч. журн. Кубан. гос. аграрного ун-та. Краснодар: ФГБОУ ВПО «Кубанский гос. аграрный ун-Т», 2015. № 109 (05). С. 1-13.

2. Иванов М.Ю. Автоматизация сетевого планирования и управления // Системы. Методы. Технологии. Братск: Братский гос. ун-т, 2013. № 2 (18). С. 63-69.

3. Киселев В.Г. Сетевые модели управления в животноводческой отрасли АПК // Управление большими системами: сб. тр. М.: Ин-т проблем управления им. В.А. Трапезникова РАН, 2011. № 32. С. $172-194$.

4. Приходько Д.О. Сетевая модель «Строй-инвест-проект» модернизированный инструмент управления процессами организации строительства и рационального использования ресурсов заказчика строительного проекта // Наука и прогресс транспорта: Вестн. Днепропетров. национального ун-та железнодорожного транспорта. Днепропетровск: Днепропетровский национальный ун-т железнодорожного транспорта им. ак. В. Лазаряна, 2010. № 33. С. 204-207.

5. Шориков А.Ф., Буценко Е.В. Экспертная система инвестиционного проектирования // Прикладная информатика. М.: Синергия Пресс, 2013. № 5 (47). С. 96-104.

6. Таха Хемди А. Введение в исследование операций. 7-е изд.: пер. с англ. М.: Издательский дом «Вильямс», 2005. 912 с. 
E.V. Butsenko, Ural State University of of Economics, Ekaterinburg, Russia. E-mail: evl@usue.ru THE PRACTICE OF USING A NETWORK OF ECONOMIC AND MATHEMATICAL MODELING OF THE PROCESS OF INVESTMENT PLANNING

Keywords: investment planning, network modeling, the parameters of the network model, schedule

The objectives of improving investment planning in today's economy can be considered as necessary components of any enterprise management process and are precisely problems. The development of technologies based on the optimization of the investment planning is highly relevant scientific problem. In this paper, we propose to use methods of network planning and management as a tool for economic and mathematical modeling of investment planning for the business entity. Appropriate to the process network model allows for the efficient organization of work on investment planning. The article describes the general methodology of the investment management process for designing an economic entity. Real example illustrates the application of the proposed network model for the development of the investment project «Start of new business in the food industry (frozen products)». The study analyzes all processes / operations and their relationships, ordering and coding work of the relevant stage of the project; built a network schedule; the parameters of the network model and built a calendar schedule, allowing time to visually see the most important process of the work. It should be noted that the network was carried out economic and mathematical modeling by two parameters - the time (duration) of the selected works in the project and their cost. The results of this study can be used by any business entity engaged in investment activities.

\section{References}

1. Zelenskaya T.M., Vanzhula D.V., Kovaleva K.A. Primenenie metodov setevogo planirovaniya i upravleniya $v$ sel'skokhozyaistvennom proizvodstve // Politematicheskii setevoi elektronyi nauchnyi zhurnal Kubanskogo gosudarstvennogo agrarnogo universiteta - Krasnodar: FGBOU VPO Kubanskii gosudarstvennyi agrarnyi universitet Publ., 2015, no.109 (05), pp. 1-13.

2. Ivanov M. Yu. Avtomatizatsiya setevogo planirovaniya $i$ upravleniya // Sistemy. Metody. Tekhnologii. - Bratsk: Bratskii gosudarstvennyi universitet Publ., 2013, no. 2 (18), pp. 63-69.

3. Kiselev V.G. Setevye modeli upravleniya $v$ zhivotnovodcheskoi otrasli APK // Upravlenie bol'shimi sistemami: sbornik trudov. - Moscow: Federal'noe gosudarstvennoe byudzhetnoe uchrezhdenie nauki Institut problem upravleniya imeni V.A. Trapeznikova RAN Publ., 2011, no. 32, pp. 172-194.

4. Prikhod'ko D.O. Setevaya model' «Stroi-invest-proekt» modernizirovannyi instrument upravleniya protsessami organizatsii stroitel'stva i ratsional'nogo ispol'zovaniya resursov zakazchika stroitel'nogo proekta // Nauka i progress transporta. Vestnik dnepropetrovskogo natsional'nogo universiteta zheleznodorozhnogo transporta - Dnepropetrovsk: Dnepropetrovskii natsional'nyi universitet zheleznodorozhnogo transporta im. ak. V. Lazaryana Publ., 2010, no. 33, pp. 204-207.

5. Shorikov A.F., Butsenko E.V. Ekspertnaya sistema investitsionnogo proektirovaniya [Expert system of investment projecting]. Prikladnaya informatika - Journal of Applied Informatics, 2013, no. 5, pp. 96-104.

6. Taha Hemdy A. Operations Research: An Introduction. 7th ed. Prentice Hall Publ., 2002. 848 p. (Russ. Ed.: Takha Khemdi A. Vvedenie v issledovanie operatsii. Moscow, Vil'yams Publ., 2005. 912 p.). 